\title{
Blood pressure levels in patients with subclinical thyroid dysfunction: a meta-analysis of cross-sectional data
}

\author{
Yunfei Cai ${ }^{1,2}$, Yongkui Ren ${ }^{3}$ and Jingpu Shi ${ }^{1,2}$
}

The relationship between subclinical thyroid dysfunction and blood pressure is controversial and has received insufficient attention. The aim of this study was to assess whether blood pressure levels in patients with subclinical thyroid dysfunction differ from those of euthyroid subjects. A meta-analysis of all cross-sectional studies was performed to compare the blood pressure levels in patients with subclinical thyroid dysfunction with those of healthy controls. A computer-based online retrieval of databases (MEDLINE and EMBASE) and manual searches were undertaken to identify articles that addressed the association between subclinical thyroid dysfunction and blood pressure levels and were published through 2010, using no language restrictions. The meta-analysis was performed using STATA 11 (Stata). Seven cross-sectional studies were examined. In patients with subclinical hypothyroidism, the pooled estimate of the weighted mean difference (WMD) of increased blood pressure revealed a significant difference in both systolic blood pressure (SBP; WMD with $95 \%$ confidence interval (Cl) $1.89 \mathrm{~mm} \mathrm{Hg}$ $(0.98-2.80), P<0.05)$ and diastolic blood pressure (DBP; WMD with $95 \% \mathrm{Cl} 0.75 \mathrm{~mm} \mathrm{Hg}(0.24-1.27), P<0.05)$. However, in patients with subclinical hyperthyroidism, the pooled estimate of the WMD of increased blood pressure revealed no significant difference in SBP (WMD with $95 \% \mathrm{Cl}-0.75 \mathrm{~mm} \mathrm{Hg}(-1.81$ to 0.31 )) or DBP (WMD with $95 \% \mathrm{Cl}-0.64 \mathrm{~mm} \mathrm{Hg}(-2.36$ to 1.08)). The present meta-analysis indicates that subclinical hypothyroidism is associated with increased SBP and DBP, whereas subclinical hyperthyroidism is not. Further investigation is needed to confirm blood pressure levels in patients with subclinical thyroid dysfunction.

Hypertension Research (2011) 34, 1098-1105; doi:10.1038/hr.2011.91; published online 28 July 2011

Keywords: blood pressure; hyperthyroidism; hypothyroidism; meta-analysis; thyroid disease

\section{INTRODUCTION}

Thyroid dysfunction, both hypothyroidism and hyperthyroidism, may increase the risk of hypertension. ${ }^{1,2}$ However, it is still controversial whether mild thyroid dysfunction, such as subclinical hypothyroidism and subclinical hyperthyroidism, affects blood pressure. Subjects with subclinical hypothyroidism have elevated thyroid-stimulating hormone (thyrotropin, TSH) levels and normal free thyroxine (T4) levels. ${ }^{3}$ Previous studies have suggested that subclinical abnormalities in TSH levels are associated with detrimental effects on the cardiovascular system. ${ }^{4}$ Studies have also suggested an association between subclinical hypothyroidism and hypertension, which has been subsequently confirmed by some, but not all, large cross-sectional and casecontrol studies. ${ }^{4}$ Within the past 5 years, six large cross-sectional studies on this topic have produced conflicting results. ${ }^{5-10}$ Three studies concluded that subjects with subclinical hypothyroidism had a significantly higher systolic blood pressure (SBP) than euthyroid subjects (age, sex and body mass index adjusted). ${ }^{5,9,10}$ However, discrepancies between study results may have arisen from differences in the level of adjustment for other cardiovascular risk factors among the included studies.

Subjects with subclinical hyperthyroidism have low serum TSH levels and normal free thyroid hormone (free T4) levels. The consequences of subclinical hyperthyroidism have been less frequently studied than those of subclinical hypothyroidism. Subclinical hyperthyroidism has been associated with decreased blood pressure level, ${ }^{9}$ but with conflicting data. $5,7,10,11$

There have been no analyses performed to date on the relationship between subclinical thyroid dysfunction and blood pressure. To determine whether subclinical thyroid dysfunction is associated with increased blood pressure, we performed a meta-analysis of crosssectional studies that examined the relationship between subclinical thyroid dysfunction and the risk of increased blood pressure levels.

${ }^{1}$ Department of Clinical Epidemiology, The First Affiliated Hospital, China Medical University, Shenyang, China; ${ }^{2}$ Center of Evidence Based Medicine, The First Affiliated Hospital, China Medical University, Shenyang, China and ${ }^{3}$ Department of Cardiology, The First Affiliated Hospital, China Medical University, Shenyang, China

Correspondence: Dr J Shi, Department of Clinical Epidemiology, the First Affiliated Hospital, China Medical University, No. 155 Nanjing Bei Street, Heping District, Shenyang, Liaoning Province 110001, China.

E-mail: jingpusepi@yahoo.com

Received 6 December 2010; revised 30 March 2011; accepted 10 April 2011; published online 28 July 2011 


\section{METHODS}

Meta-analyses of observational studies present particular challenges because of inherent biases and differences in study designs. ${ }^{12}$ To ensure objectivity, we conducted and reported this analysis according to the guidelines of the Meta-Analysis of Observational Studies in Epidemiology Group. ${ }^{13}$

\section{Search strategy}

Searches were conducted in electronic databases MEDLINE and EMBASE through 2010 to identify articles that reported blood pressure values in patients with subclinical thyroid dysfunction compared with euthyroid subjects. Manual searches of key journals and abstracts from major annual meetings were conducted in the fields of endocrinology and cardiovascular disease in an effort to identify relevant unpublished data. Additional unpublished studies were also sought from web sites. The search strategy included the following: the Medical Subject Headings term 'Hypothyroidism'[Mesh] or 'Hyperthyroidism'[Mesh] or 'Thyroid Disease'[Mesh] or 'Thyrotropin'[Mesh] and the text word terms 'subclinical hypothyroidism' or 'subclinical hyperthyroidism' or 'subclinical thyroid dysfunction' or 'thyroid-stimulating hormone'; and the Medical Subject Headings terms 'Blood Pressure'[Mesh] or 'Hypertension' [Mesh] and the text word terms 'systolic blood pressure' or 'diastolic blood pressure. No language restrictions were used in the search.

\section{Inclusion criteria}

Serum thyrotropin and free thyroxine concentrations were measured with reference ranges as follows. For thyrotropin levels, the normal value for the lower limit of the range was $0.25-0.4 \mathrm{mUl}^{-1}$, and the upper limit was $3.8-4.8 \mathrm{mUl}^{-1}$. The normal free $\mathrm{T} 4$ range of $0.6-1.9 \mathrm{ng} \mathrm{dl}^{-1}$ was used in most studies. ${ }^{5-11}$ The TSH reference range is a topic of debate; however, TSH levels are critically important in diagnosing subclinical disease. Subclinical hypothyroidism is defined as the presence of an elevated TSH and a normal T4 level. ${ }^{3}$ Several reviews suggest a TSH upper limit cutoff of $4.5-5.0 \mathrm{mUl}^{-1}, 3,14$ but some authors suggest that the upper limit of the TSH range should be reduced to 2.5-3.0 $\mathrm{mU} \mathrm{l}^{-1},{ }^{15,16}$ based on a higher rate of progression to overt hypothyroidism and a higher prevalence of antithyroid antibodies than in euthyroid subjects. ${ }^{17}$ In the absence of a consensus, we did not specify a TSH cutoff to define subclinical hypothyroidism; the TSH cutoff value of each included study is listed in Table 2. Similarly, we did not specify a TSH range for subclinical hyperthyroidism, but all studies had a cutoff value close to 0.25 to $0.4 \mathrm{mUl}^{-1}$ (values listed in Table 3). Studies were included if they met the following criteria: (1) analyses were reported for two groups defined according to the thyroid function test results as either subclinical hypothyroidism or subclinical hyperthyroidism; (2) reporting of age-, sex- and body mass index-adjusted SBP and diastolic BP (DBP) data were available for patients with subclinical hyperthyroidism or subclinical hypothyroidism and compared with the euthyroid population; and (3) reporting of s.d., s.e. or $95 \%$ confidence intervals (CIs) for the mean SBP and DBP. This information was necessary for the quantitative analyses. Only studies where age and sex were similar between the two patient groups were included in the meta-analysis.

\section{Exclusion criteria}

Participants who were taking medicines that influence thyroid function or were undergoing antihypertensive treatment were excluded. Participants with overt thyroid dysfunction were also excluded. ${ }^{5}$ Participants with a history of thyroid disease and those who were taking medication affecting thyroid function, ${ }^{6,7}$ those who were diagnosed with hypothyroidism or hyperthyroidism in this investigation as well as participants with hypertension who had taken oral antihypertensive drugs in the previous 4 weeks were also excluded. ${ }^{6}$ Participants with any thyroid disease ${ }^{8}$ and who were being treated for thyroid disease and hypertension were also excluded. ${ }^{9}$ Subjects with overt hyperthyroidism or increased serum thyrotropin levels who were receiving antihypertensive medications were also excluded. ${ }^{11}$ Researchers were unable to exclude subjects who were on a drug treatment that might affect measurements of thyroid function, but the number of participants on such therapy was expected to be small. ${ }^{10}$

\section{Study selection and data extraction}

Two reviewers (CYF and RYK) independently screened the abstracts and titles of the search results, and articles were eliminated only if they did not investigate the association between subclinical thyroid dysfunction and blood pressure levels. The same two reviewers independently evaluated studies for eligibility on the basis of the predefined set of eligibility criteria.

Information from each study was extracted independently by the two reviewers (CYF and RYK), using a standardized data extraction form. General characteristics of the study (author, year of publication, country, time of study, study design, exclusion criteria, TSH assay, sphygmomanometer, position, reading(s) used and adjusted factors), characteristics of the subclinical thyroid dysfunction and euthyroid groups (age, sex, body mass index and TSH levels) and results (sample size, SBP mean \pm s.d., DBP mean \pm s.d.) were recorded, where available, and double checked. Discrepancies in data extraction between reviewers were resolved by discussion.

\section{Statistical analysis}

SBP and DBP levels in each study were extracted as mean \pm s.d. When not reported, missing mean differences and s.d.'s were estimated based on reported CIs, and the authors of the study were contacted if necessary. The s.e.m. was transformed into s.d. weighted mean difference (WMD) and 95\% CIs in SBP and DBP were calculated for all eligible studies in the meta-analysis and combined using fixed or random-effects models ${ }^{18}$ where appropriate. Heterogeneity between the results of different studies was examined using $\chi^{2}$ tests for significance (a $P$-value $<0.1$ was considered to be statistically significant) and $I^{2}$ tests $\left(I^{2}>50 \%\right.$ : significant heterogeneity; $I^{2}<25 \%$ : insignificant heterogeneity). ${ }^{19}$ To assess the extent of publication bias, Begg's correlation and Egger's regression for publication bias were used..$^{20,21}$ Visual inspection of publication bias using a funnel plot of the data was not employed because bias may be incorrectly inferred if studies are heterogeneous. ${ }^{22}$ Sensitivity analysis was conduced for all studies except those with borderline eligibility. Meta-analysis was conducted using STATA 11 for Windows (Stata, College Station, TX, USA).

\section{RESULTS}

\section{Search results}

Our review yielded seven large cross-sectional studies ${ }^{5-11}$ (including subclinical hypothyroidism and subclinical hyperthyroidism). These studies reported the number of patients with a diagnosis of subclinical thyroid dysfunction at baseline. Of the 255 reports (including electronic and manual retrieval) initially identified, we excluded 231 studies that did not investigate the association between subclinical thyroid dysfunction and blood pressure. Of the 24 articles selected for detailed evaluation, 4 studies did not assess the risk of high blood pressure in subjects with subclinical thyroid dysfunction, 4 studies contained participants with a personal history of thyroid disease or participants who were taking medication that affects thyroid function and 2 studies did not provide risk estimates and CIs or the information necessary to calculate them from the report. Six low-quality case-control studies were excluded because they did not report blood pressure measurements. When similar data were published twice, we included the article with the most definitive and extractable information; this led to the exclusion of one study. ${ }^{23}$ Seven cross-sectional studies remained and were included in the meta-analysis. Table 1 shows the characteristics of all seven studies included in our analysis. Tables 2 and 3 show the patient characteristics based on risk factors and outcomes for subclinical hypothyroidism and subclinical hyperthyroidism, respectively.

\section{SBP in subclinical hypothyroidism and cumulative meta-analysis} Six studies ${ }^{5-10}$ reported the SBP levels of patients with subclinical hypothyroidism and euthyroid subjects. No statistical heterogeneity was evident among the studies $\left(P=0.225 ; I^{2}=27.9 \%\right)$, and hence WMD was pooled using a fixed-effect model. Three studies ${ }^{5,9,10}$ out of the six concluded that SBP levels were substantially higher in the 


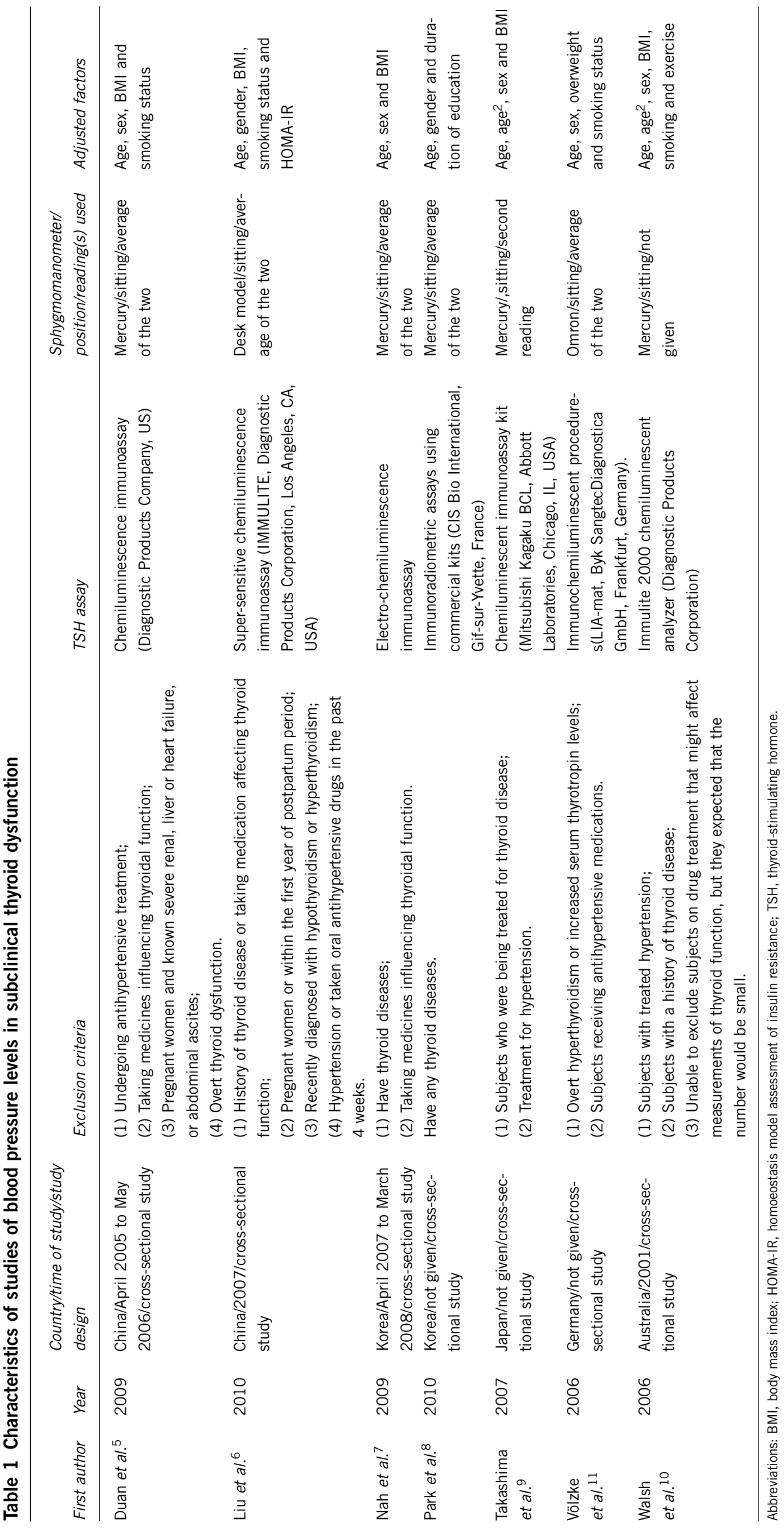



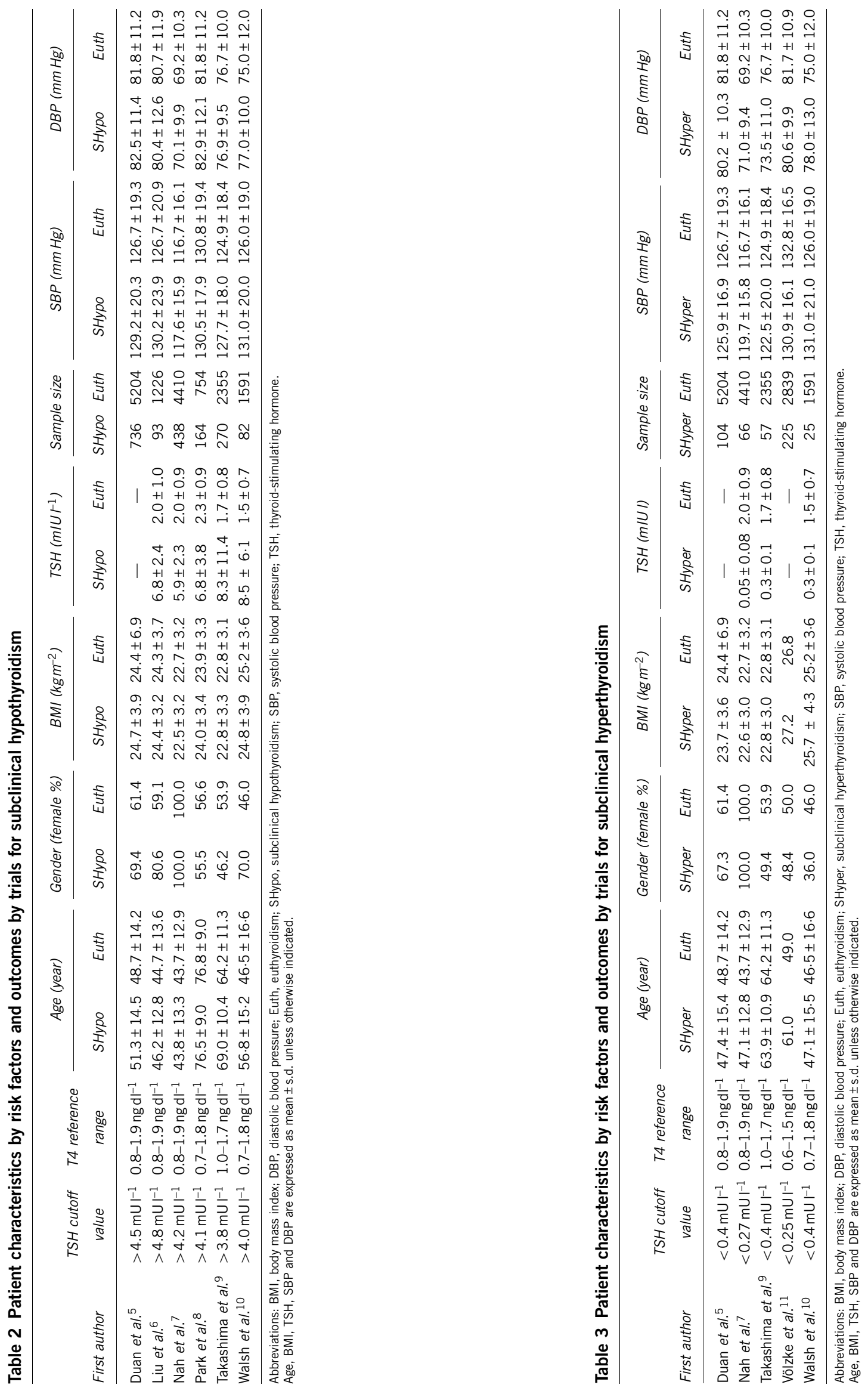
group with subclinical hypothyroidism compared with controls (WMD with 95\% CI $1.89 \mathrm{~mm} \mathrm{Hg}(0.98-2.80), P=0.000$; Figure 1). Because two studies ${ }^{7,8}$ did not specify the exclusion of participants with hypertension who had taken oral antihypertensive drugs, we did a sensitivity analysis excluding the two studies that showed a pooled WMD of $2.81 \mathrm{~mm} \mathrm{Hg}$ (95\% CI 1.61-4.00, $P=0.000$ ). Exclusion of the two studies did not yield results that were similar to the previous pooled WMD. However, sensitivity analysis showed a higher blood pressure level in patients with subclinical hypothyroidism compared with those with euthyroidism, which indicated that the exclusion of the two studies from our meta-analysis strengthened the estimate. Before and after the sensitivity analysis, SBP in subjects with subclinical hypothyroidism was higher than that of the euthyroid group $(P<0.05$ for both). Sequential cumulative meta-analyzed results for each year beginning with 2006 were calculated. From 2009 onward, the pooled cumulative WMD varied between 2.10 and 2.15 with no evident trend. Neither the Egger's regression asymmetry test $(P=0.507)$ nor the Begg's adjusted rank correlation test $(P=1.000)$ regarding SBP in subclinical hypothyroidism indicated the existence of publication bias.

DBP in subclinical hypothyroidism and cumulative meta-analysis Six studies ${ }^{5-10}$ evaluated DBP levels in patients with subclinical hypothyroidism. We pooled the six studies as a combined analysis of DBP using the fixed-effects method because no statistical heterogeneity was evident among studies $\left(P=0.728 ; I^{2}=0.0 \%\right)$. The pooled estimate, after adjustment for variables mentioned in Table 1 , represented a significant increase in blood pressure levels among subjects with subclinical hypothyroidism compared with controls (WMD with 95\% CI $0.75 \mathrm{~mm} \mathrm{Hg}$ (0.24-1.27), $P=0.004$; Figure 2). Because two

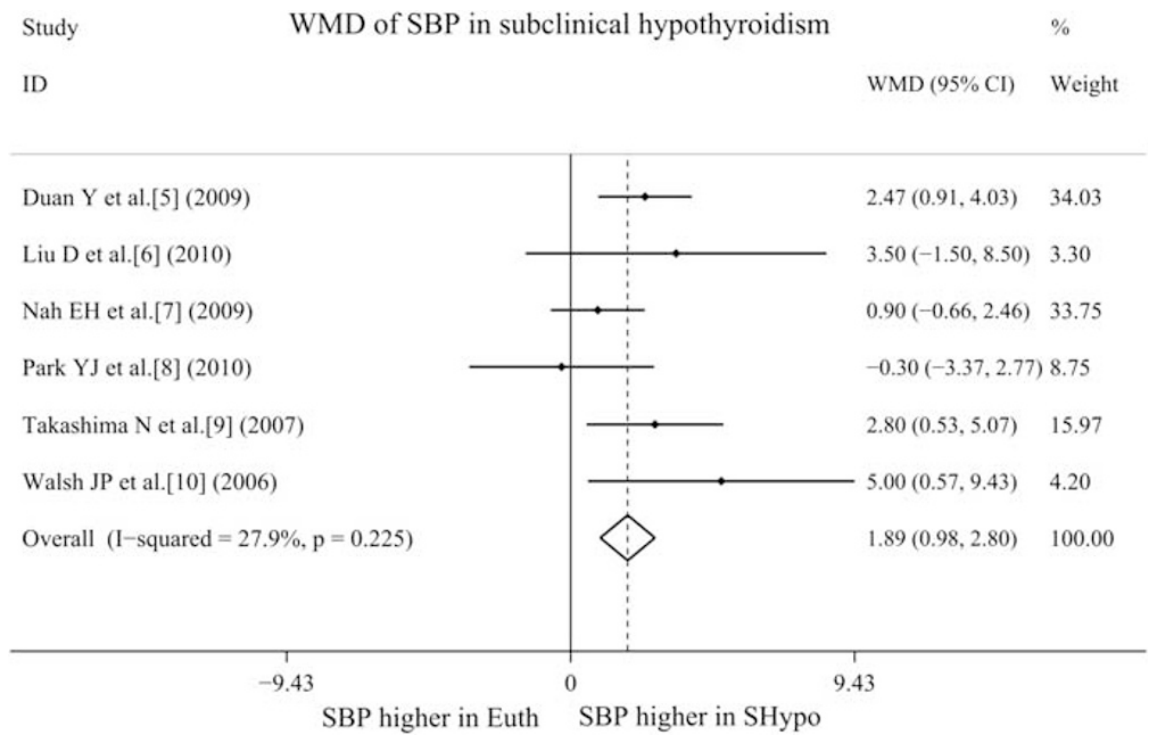

Figure 1 Comparison of systolic blood pressure (SBP) in subclinical hypothyroidism and euthyroidism in six cross-sectional studies. Cl, confidence interval; Euth, euthyroidism, SHypo, subclinical hypothyroidism; WMD, weighted mean difference.

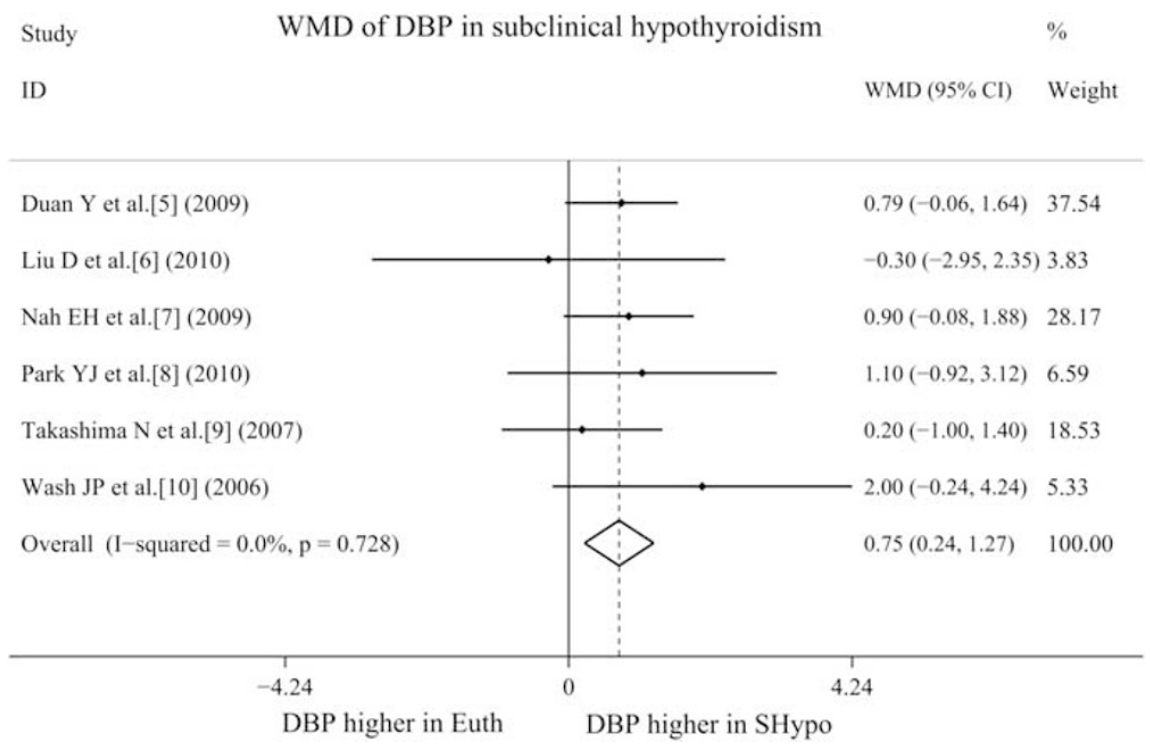

Figure 2 Comparison of diastolic blood pressure (DBP) in subclinical hypothyroidism and euthyroidism in six cross-sectional studies. Cl, confidence interval; Euth, euthyroidism, SHypo, subclinical hypothyroidism; WMD, weighted mean difference. 
studies $^{7,8}$ did not report whether participants with hypertension who had taken oral antihypertensive drugs were excluded, we did a sensitivity analysis excluding the two studies that showed a pooled WMD of $0.66 \mathrm{~mm} \mathrm{Hg}$ (95\% CI 0.02-1.03), and the exclusion of the two studies yielded similar results. Sequential cumulative meta-analyzed results for each year beginning with 2006 were calculated. From 2009 onward, the pooled cumulative WMD varied between 0.88 and 0.94 , with no evident trend. Neither the Egger's regression asymmetry test $(P=0.896)$ nor the Begg's adjusted rank correlation test $(P=0.707)$ regarding DBP in subclinical hypothyroidism indicated the existence of publication bias.

SBP in subclinical hyperthyroidism and cumulative meta-analysis We performed both a fixed-effect meta-analysis and a cumulative meta-analysis because no statistical heterogeneity was evident among the studies $\left(P=0.138 ; I^{2}=42.5 \%\right)$. In the five studies ${ }^{5,7,9-11}$ that reported SBP levels, the WMD of increased blood pressure in patients with subclinical hyperthyroidism revealed no significant difference (WMD with $95 \% \mathrm{CI}-0.75 \mathrm{~mm} \mathrm{Hg}(-1.81$ to 0.31$)$ ). Because one study $^{7}$ did not report whether they excluded participants with hypertension who had taken oral antihypertensive drugs, we performed a sensitivity analysis excluding the study that showed a pooled WMD of $-1.06 \mathrm{~mm} \mathrm{Hg}$ (95\% CI -2.16 to 0.04$)$. The exclusion of that study yielded similar results. Figure 3 displays the pooled cumulative WMD from 2006 to 2009, which ranged from 5.00 to $-0.75 \mathrm{~mm} \mathrm{Hg}$. From 2006 onward, the pooled cumulative WMD varied between -1.58 and -0.75 , with no evident trend. Neither the Egger's regression asymmetry test $(P=0.411)$ nor the Begg's adjusted rank correlation test $(P=0.806)$ regarding SBP in subclinical hyperthyroidism indicated the existence of publication bias.

DBP in subclinical hyperthyroidism and cumulative meta-analysis There were five studies ${ }^{5,7-11}$ that evaluated DBP levels in those with subclinical hyperthyroidism. Significant statistical heterogeneity was evident among the studies $\left(P=0.030 ; I^{2}=62.6 \%\right)$; therefore, WMD was pooled using a random-effect model. The WMD of DBP in patients with subclinical hyperthyroidism revealed no significant difference (WMD with $95 \% \mathrm{CI}-0.64 \mathrm{~mm} \mathrm{Hg}$ ( -2.36 to 1.08$)$ ).
Because one study ${ }^{7}$ did not report whether they excluded participants with hypertension who had taken oral antihypertensive drugs, we performed a sensitivity analysis excluding the study that showed a pooled WMD of $-1.36 \mathrm{~mm} \mathrm{Hg}$ (95\% CI -2.39 to 0.34$)$. The exclusion of that study did not significantly modify the overall effects observed. Sequential cumulative meta-analyzed results for each year from 2006 were calculated. From 2006 onward, the pooled cumulative WMD varied between -0.59 and -1.24 , with no evident trend. Neither the Egger's regression asymmetry test $(P=0.604)$ nor the Begg's adjusted rank correlation test $(P=1.000)$ regarding DBP in subclinical hyperthyroidism indicated the existence of publication bias.

\section{DISCUSSION}

Our findings are based on the information available on subclinical thyroid dysfunction and blood pressure levels. When possible, we quantitatively estimated the association between this thyroid condition and increased blood pressure.

The results of our study suggest an association between subclinical hypothyroidism and increased blood pressure levels. Several mechanisms could explain why subclinical hypothyroidism has an adverse effect on blood pressure. Clinical hypothyroidism is known to increase blood pressure levels, and the main underlying cause of this is thought to be the degree of systemic vascular resistance present in patients with clinical hypothyroidism. T3 can directly act on arterial smooth muscle cells of blood vessels to cause vasodilation. ${ }^{24}$ In hypothyroidism, declining T3 levels result in an increased vascular resistance, causing an increase in blood pressure. Hypothyroidism can also lead to abnormal sodium metabolism, increased sympathetic nervous system activity and a decreased glomerular filtration rate, which may contribute to the development of hypertension. ${ }^{25-27}$

Blood pressure salt sensitivity was also one of the critical factors for hypertension in hypothyroid patients. ${ }^{28}$ The study by Gumieniak et l. $^{25}$ showed that the relationship between free thyroxine index (FTI) and salt sensitivity is partly mediated by the vascular action of the thyroid hormone. Recent studies indicate that cardiovascular disorders have existed in the subclinical hypothyroidism stage. Luboshitzky et al..$^{29}$ found that the prevalence of hypertension in the

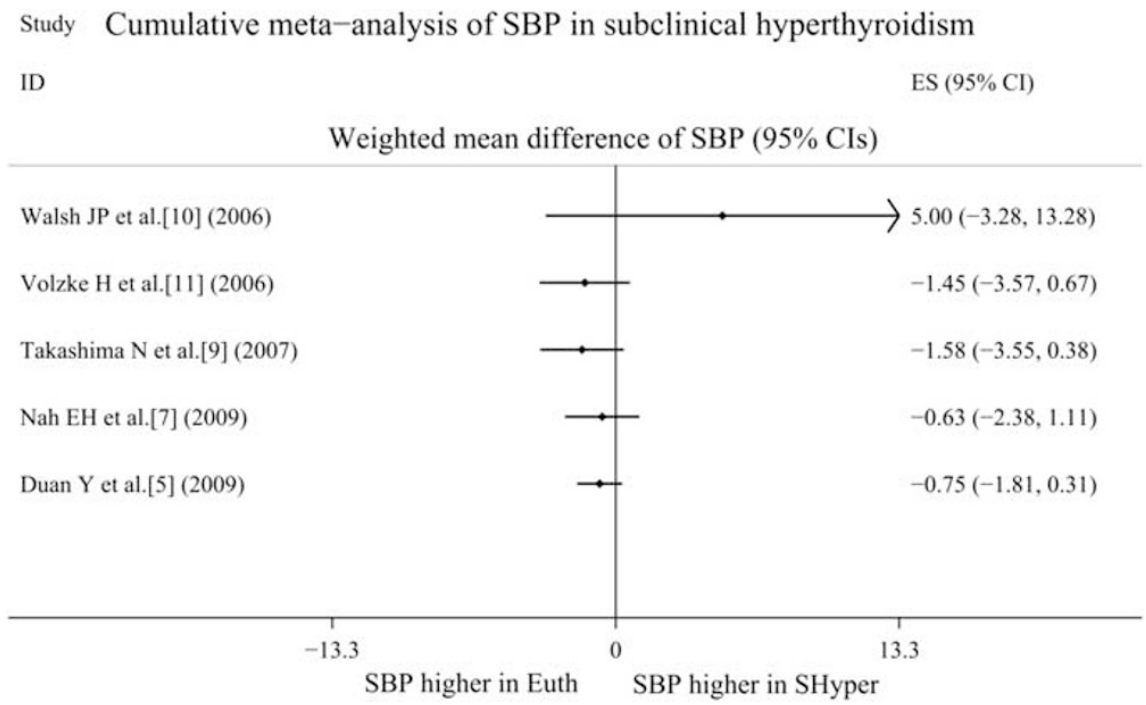

Figure 3 Cumulative meta-analysis of systolic blood pressure (SBP) comparing subclinical hyperthyroidism with euthyroidism in five cross-sectional studies, 2006-2009. Cl, confidence interval; Euth, euthyroidism; SHyper, subclinical hyperthyroidism. 
subclinical hypothyroidism group was significantly higher than that in the normal control group, supporting our conclusion. Rotterdam's study showed that subclinical hypothyroidism was an independent risk factor for atherosclerosis and myocardial infarction. ${ }^{30}$ Blood hypercoagulability, blood viscosity and lipid abnormalities presenting in subclinical hypothyroidism patients could increase the risk for atherosclerosis. ${ }^{30}$ These factors may also be involved in the pathogenesis in which subclinical hypothyroidism affects blood pressure.

In the study by Liu et al., ${ }^{6}$ their investigation confirmed that subclinical hypothyroidism could increase the risk for hypertension. These results differ from those of the cross-sectional study by Duan et al..$^{5}$ and Walsh et al. ${ }^{10}$ in which subclinical hypothyroidism was not associated with an increase in blood pressure. This lack of association may reflect the smaller number of subjects in the case-control studies or selection bias that arose from the clinic-based design in the study by Walsh et al. ${ }^{10}$ Other possibilities for this discrepancy include differences in race, lifestyle and genetic background of the sampled population. No compelling explanation has been widely accepted.

The present study addressed possible associations between subclinical hyperthyroidism and blood pressure. The present meta-analyses did not reveal an increased blood pressure in individuals with decreased or suppressed serum thyrotropin levels and free thyroid hormones within the reference range. In the study by Völzke et al., ${ }^{11}$ a community-based cross-sectional study was conducted among 4087 subjects in West Pomerania, the northeast area of Germany; they concluded that subclinical hyperthyroidism is not associated with hypertension. In contrast, Walsh et al. ${ }^{10}$ conducted a communitybased study in Australia and found that the prevalence of hypertension was higher in patients with subclinical hyperthyroidism than that in the group with euthyroidism. Indeed, another study conducted by the same team disclosed that subclinical hyperthyroidism was not significantly related to adverse cardiovascular diseases. ${ }^{31}$ This suggests that the significant difference in hypertension rates between groups with subclinical hyperthyroidism and euthyroidism was probably a chance finding.

The etiologies in the subclinical hypothyroidism group are not specified, but likely represent several clinical situations, which most commonly involve (1) early, mild primary hypothyroidism, such as in Hashimoto's thyroiditis, (2) some cases of acute non-thyroidal illness and (3) the recovery phase of some patients after non-thyroidal illnesses. Subclinical hypothyroidism is most commonly caused (50-80\% of cases) by chronic autoimmune thyroiditis, which is typically characterized by hypoechogenicity on ultrasound thyroid examination and by the presence of thyroid autoantibodies in the serum; these antibodies are most commonly thyroperoxidase antibodies and are less commonly thyroglobulin antibodies, and rarely TSH-receptor blocking antibodies. ${ }^{32}$ Less frequently, subclinical hypothyroidism is because of other conditions; for example, iodine intake is one of the causes for subclinical hypothyroidism. Unfortunately, the studies included in this meta-analysis did not report the iodine intake in each of these patients.

Although our statistical analyses showed that publication bias was unlikely, it cannot be excluded because the capacity to detect publication bias is reduced when meta-analyses are based on a limited number of studies. ${ }^{20,21}$ The major limitation of our analysis was that the definitions of subclinical hypo- and hyper-thyroidism were slightly different between studies; the different TSH cutoffs used reflect the absence of consensus about how to define subclinical hypothyroidism. ${ }^{3,14-16}$ Some studies have a TSH cutoff of $<4.5 \mathrm{mUl}^{-1}$. ${ }^{7-10}$ The inclusion of subjects with an almost normal TSH value would be expected to skew the effects of any observed associations because some of these subjects may not have subclinical hypothyroidism. However, TSH concentrations are not identical when measured by assays from various kits used in different countries. This may be the cause of the observed variation for TSH cutoffs. Thus, an international standardization of thyroid function testing, currently under development, is needed for future studies. Another limitation of our study includes the lack of individual patient data, precluding further exploration of the effect of individual differences on the association between subclinical thyroid dysfunction and higher blood pressure among men and women. Additionally, information on menopausal status and hormone replacement therapy use was not available for the present analysis; therefore, we could not exclude their potential confounding effects. In addition, we did not have information on the duration of this subclinical state or any data on the progression or treatment of thyroid dysfunction. The natural history of subclinical hypothyroidism is variable; thyroid function normalizes spontaneously in some subjects, whereas it progresses to overt hypothyroidism in others. It is therefore possible that the increase in cardiac events observed in the subclinical hypothyroidism group arose because of a progression to overt hypothyroidism rather than simply because of the subclinical hypothyroidism. Finally, any meta-analysis contains its inherent limitations because it is based on the summary of previous published sources and is limited by many factors; thus, cautious interpretation of our results is recommended.

\section{CONCLUSIONS}

Our meta-analysis indicates that subclinical hypothyroidism is associated with increased SBP and DBP. However, subclinical hyperthyroidism is not associated with increased SBP or DBP. Additional prospective randomized studies are necessary to evaluate these observations.

\section{CONFLICT OF INTEREST}

The authors declare no conflict of interest.

\section{ACKNOWLEDGEMENTS}

The authors thank all the participants in this study.

\section{DISCLOSURE}

Neither the entire paper nor any part its content has been published or accepted elsewhere. It is not being submitted to any other journal.

1 Cappola AR, Ladenson PW. Hypothyroidism and atherosclerosis. J Clin Endocrinol Metab 2003; 88: 2438-2444.

2 Prisant LM, Gujral JS, Mulloy AL. Hyperthyroidism: a secondary cause of isolated systolic hypertension. J Clin Hypertens (Greenwich) 2006; 8: 596-599.

3 Helfand M. Screening for subclinical thyroid dysfunction in nonpregnant adults: a summary of the evidence for the U.S. Preventive Services Task Force. Ann Intern Med 2004; 140: 128-141.

4 Monzani F, Dardano A, Caraccio N. Does treating subclinical hypothyroidism improve markers of cardiovascular risk? Treat Endocrinol 2006; 5: 65-81.

5 Duan Y, Peng W, Wang X, Tang W, Liu X, Xu S, Mao X, Feng S, Feng Y, Qin Y, Xu K, Liu C. Community-based study of the association of subclinical thyroid dysfunction with blood pressure. Endocrine 2009; 35: 136-142.

6 Liu D, Jiang F, Shan Z, Wang B, Wang J, Lai Y, Chen Y, Li M, Liu H, Li C, Xue H, Li N, Yu J, Shi L, Bai X, Hou X, Zhu L, Lu L, Wang S, Xing Q, Teng W. A cross-sectional survey of relationship between serum TSH level and blood pressure. J Hum Hypertens 2010; 24: 134-138.

$7 \mathrm{Nah}$ EH, Lee JG. [The relationship between thyroid function and the risk factors of cardiovascular disease at female medical checkups]. Korean J Lab Med 2009; 29 . 286-292.

8 Park YJ, Lee EJ, Lee YJ, Choi SH, Park JH, Lee SB, Lim S, Lee WW, Jang HC, Cho BY, Woo JI, Kim KW. Subclinical hypothyroidism $(\mathrm{SCH})$ is not associated with metabolic derangement, cognitive impairment, depression or poor quality of life $(\mathrm{Q} o \mathrm{~L})$ in elderly subjects. Arch Gerontol Geriatr 2010; 50: e68-e73. 
9 Takashima N, Niwa Y, Mannami T, Tomoike H, Iwai N. Characterization of subclinical thyroid dysfunction from cardiovascular and metabolic viewpoints: the Suita study. Circ J 2007; 71: 191-195.

10 Walsh JP, Bremner AP, Bulsara MK, O'Leary P, Leedman PJ, Feddema P, Michelangeli V. Subclinical thyroid dysfunction and blood pressure: a community-based study. Clin Endocrinol (Oxf) 2006; 65: 486-491.

11 Volzke H, Alte D, Dorr M, Wallaschofski H, John U, Felix SB, Rettig R. The association between subclinical hyperthyroidism and blood pressure in a population-based study. J Hypertens 2006; 24: 1947-1953.

12 Huston P, Naylor CD. Health services research: reporting on studies using secondary data sources. CMAJ 1996; 155: 1697-1709.

13 Stroup DF, Berlin JA, Morton SC, Olkin I, Williamson GD, Rennie D, Moher D, Becker BJ, Sipe TA, Thacker SB. Meta-analysis of observational studies in epidemiology: a proposal for reporting. Meta-analysis Of Observational Studies in Epidemiology (MOOSE) group. JAMA 2000; 283: 2008-2012.

14 Surks MI, Ortiz E, Daniels GH, Sawin CT, Col NF, Cobin RH, Franklyn JA, Hershman JM, Burman KD, Denke MA, Gorman C, Cooper RS, Weissman NJ. Subclinical thyroid disease: scientific review and guidelines for diagnosis and management. JAMA 2004; 291: 228-238.

15 Haugen B. When Isn't the TSH Normal and Why? Clinical Implications and Causes Paper presented at the 12th Annual Meeting of the American Association of Clinical Endocrinologists (AACE) 2003, San Diego, CA.

16 Lee S. When Is the TSH Normal? New Criteria for Diagnosis and Management. Paper presented at the 12th Annual Meeting of the American Association of Clinical Endocrinologists (AACE) 2003, San Diego, CA.

17 Vanderpump MP, Tunbridge WM, French JM, Appleton D, Bates D, Clark F, Grimley Evans J, Hasan DM, Rodgers H, Tunbridge F. The incidence of thyroid disorders in the community: a twenty-year follow-up of the Whickham Survey. Clin Endocrinol (Oxf) 1995; 43: 55-68.

18 DerSimonian R, Laird N. Meta-analysis in clinical trials. Control Clin Trials 1986; 7: $177-188$.

19 Higgins JP, Thompson SG, Deeks JJ, Altman DG. Measuring inconsistency in metaanalyses. BMJ 2003; 327: 557-560.
20 Begg CB, Mazumdar M. Operating characteristics of a rank correlation test for publication bias. Biometrics 1994; 50: 1088-1101.

21 Egger M, Davey Smith G, Schneider M, Minder C. Bias in meta-analysis detected by a simple, graphical test. BMJ 1997; 315: 629-634.

22 Terrin N, Schmid CH, Lau J, Olkin I. Adjusting for publication bias in the presence of heterogeneity. Stat Med 2003; 22: 2113-2126.

23 Duan Y, Wang X, Peng W, Feng Y, Tang W, Wu X, Mao X, Bo R, Li W, Chen J, Qin Y, Liu C. Gender-specific associations between subclinical hypothyroidism and blood pressure in Chinese adults. Endocrine 2009; 36: 438-444.

24 Ojamaa K, Klemperer JD, Klein I. Acute effects of thyroid hormone on vascular smooth muscle. Thyroid 1996; 6: 505-512.

25 Gumieniak O, Perlstein TS, Hopkins PN, Brown NJ, Murphey LJ, Jeunemaitre X, Hollenberg NK, Williams GH. Thyroid function and blood pressure homeostasis in euthyroid subjects. J Clin Endocrinol Metab 2004; 89: 3455-3461.

26 Montenegro J, Gonzalez O, Saracho R, Aguirre R, Martinez I. Changes in renal function in primary hypothyroidism. Am J Kidney Dis 1996; 27: 195-198.

27 Myrup B, Bregengard C, Faber J. Primary haemostasis in thyroid disease. J Intern Med 1995; 238: 59-63.

28 Marcisz C, Jonderko G, Kucharz EJ. Influence of short-time application of a low sodium diet on blood pressure in patients with hyperthyroidism or hypothyroidism during therapy. Am J Hypertens 2001; 14: 995-1002.

29 Luboshitzky R, Aviv A, Herer P, Lavie L. Risk factors for cardiovascular disease in women with subclinical hypothyroidism. Thyroid 2002; 12: 421-425.

30 Saltiki K, Voidonikola P, Stamatelopoulos K, Mantzou E, Papamichael C, Alevizaki M. Association of thyroid function with arterial pressure in normotensive and hypertensive euthyroid individuals: a cross-sectional study. Thyroid Res 2008; 1: 3.

31 Walsh JP, Bremner AP, Bulsara MK, O'Leary P, Leedman PJ, Feddema P, Michelangeli V. Subclinical thyroid dysfunction as a risk factor for cardiovascular disease. Arch Intern Med 2005; 165: 2467-2472.

32 Papi G, Uberti ED, Betterle C, Carani C, Pearce EN, Braverman LE, Roti E. Subclinical hypothyroidism. Curr Opin Endocrinol Diabetes Obes 2007; 14: 197-208. 